\section{G. Avila-Ortiz * , S. Elangovan', K.W.O. Kramer ${ }^{2,3}$, D. Blanchette ${ }^{2}$, and D.V. Dawson ${ }^{2}$}

${ }^{1}$ Department of Periodontics, The University of Iowa, Iowa City, IA, USA; ${ }^{2}$ Division of Biostatistics and Research Design, Dows Institute for Dental Research, The University of Iowa, Iowa City, IA, USA; and ${ }^{3}$ Health Integrity, LLC, Easton, MD, USA; *corresponding author, gustavo-avila@uiowa.edu

J Dent Res 93(10):950-958, 2014

\title{
Effect of Alveolar Ridge Preservation after Tooth Extraction: A Systematic Review and Meta-analysis
}

\section{INTRODUCTION}

$\mathbf{E}^{\mathrm{x}}$ xtraction is generally indicated when a tooth cannot be restored or maintained in acceptable conditions for long-term health, function, and/or esthetics. Tooth loss has a direct impact on quality of life by impairing the ability to masticate, speak, and, in some instances, socialize (Gerritsen et al., 2010). Additionally, the absence of a tooth in its alveolus triggers a cascade of biological events that typically result in significant local anatomic changes (Van der Weijden et al., 2009). Preclinical and clinical studies have demonstrated that alveolar ridge volume loss postextraction is an irreversible process that involves both horizontal and vertical reduction (Schropp et al., 2003; Araujo and Lindhe, 2009). Alveolar ridge atrophy may have a considerable impact on tooth replacement therapy, particularly when implant-supported restorations are planned (Seibert and Salama, 1996). Therefore, alveolar ridge preservation (ARP) has become a key component of contemporary clinical dentistry.

Historically, the first therapeutic attempts to prevent alveolar ridge resorption were performed by root retention, with the primary goal of maximizing the stability of removable prostheses (Osburn, 1974). Nevertheless, root retention is not always feasible because of fracture, caries, and/or strategic reasons. ARP via "socket grafting" emerged in the mid-1980s as a therapeutic alternative to root submergence. Its use was rationalized on the notion that "filling" the space left by the extracted tooth with a biomaterial would emulate a "root retention effect" conducive to bone preservation (Figure 1), which would subsequently facilitate endosseous implant placement by reducing the need of ancillary grafting procedures (Artzi and Nemcovsky, 1998). This approach gained popularity over the years because of its conceptual attractiveness and technical simplicity (Christensen, 1996).

Over the past 2 decades, multiple studies evaluating the efficacy of different socket-filling approaches have been conducted. In these studies, a plethora of biomaterials has been employed, including autologous bone, bone substitutes (allografts, xenografts, and alloplasts), autologous blood-derived products, and bioactive agents, among others (Darby et al., 2009). This body of knowledge contains a wealth of clinical, radiographic, and histologic outcomes. Unfortunately, the majority of studies available in the ARP literature are anecdotal case reports, case series, or inadequately powered clinical trials (Hammerle et al., 2012). Surprisingly, many of them do not report on clinically relevant outcomes, such as linear or volumetric changes, and only a few include an adequate control (i.e., undisturbed alveolus). These shortcomings are also reflected in the systematic reviews (Ten Heggeler et al., 2011; Weng et al., 2011; Horvath et al., 2012; Morjaria et al., 2014) and meta-analyses available on the topic (Vignoletti et al., 2012; Vittorini Orgeas et al., 2013), 
which present a major limitation: pooling of data from studies with marked methodological and clinical heterogeneity (e.g., single- and multirooted teeth). This raises concerns about the possibility of generating inconsistent conclusions that over- or underestimated the therapeutic potential of specific ridge preservation strategies (De Buitrago et al., 2013). Hence, there is a need for systematic reviews focused on this clinically important topic without the aforementioned limitations. The aim of this systematic review was to determine the effect of socket grafting to prevent postextraction ridge volume loss as compared to tooth extraction alone in nonmolar teeth.

\section{MATERIALS \& METHODS}

This systematic review and subsequent meta-analysis follow the guidelines of PRISMA statement (i.e., Preferred Reporting Items of Systematic Reviews and Meta-analyses; Moher et al., 2009).

\section{PICOT Question: Population, Intervention, Comparison, Outcomes, and Time}

What is the effect of ARP via socket filling following nonmolar tooth extraction compared to extraction alone in preserving the alveolar ridge dimensions, after a minimum healing time of 12 wk reported in randomized clinical trials (RCTs) in adult human subjects?

\section{Eligibility Criteria}

Searches were limited to RCTs conducted in adult human subjects. Potential studies must have recruited patients older than $18 \mathrm{yr}$ who had at least 1 nonmolar tooth extracted. Studies must have compared ARP postextraction via socket filling to untreated sockets (controls).

Additional inclusion criteria for study selection were as follows: The ridge preservation approach must have involved the utilization of a bone-grafting material (i.e., autograft, xenograft, allograft, or alloplast), covered or not with a barrier/membrane, and had a minimum healing time of $12 \mathrm{wk}$; also, alveolar ridge dimensional changes (horizontal and/or vertical) must have been assessed clinically. Studies that involved the application of any additional therapy that could have affected healing outcomes (e.g., immediate denture delivery, simultaneous soft tissue grafting, use of healing enhancers such as growth factors) were excluded. Finally, studies that used the same population as other included studies or that reviewed only the work of other investigators were excluded. The literature search and selection protocol are in the Appendix.

\section{Outcome Measures}

The outcome measures of interest in this systematic review were mean ridge dimensional changes-horizontal (buccolingual) and vertical (midbuccal, midlingual, mesial, distal) - from baseline (tooth extraction) to final assessment (minimum of $12 \mathrm{wk}$ ).

\section{Data Extraction}

Two reviewers (G.A.-O. and S.E.) independently extracted the data using an abstraction form. In the instance of inconsistencies in data extraction as observed by the arbiter (K.W.O.K.), spe-

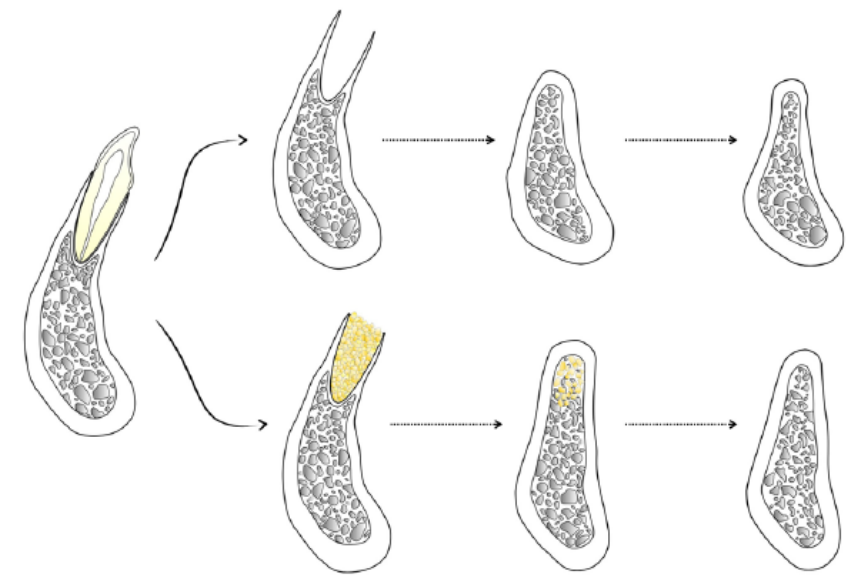

Figure 1. Illustration comparing expected alveolar bone changes after natural healing (upper row) as compared with alveolar ridge preservation via socket grafting (lower row) after tooth extraction.

cific items were referred back to the 2 reviewers for confirmation and reabstraction. Any unsettled discrepancies were resolved by consensus involving all the authors.

Data collected from each trial included (1) study and participant characteristics (year of publication, country where it was conducted, number of groups, number of subjects in each group, age of subjects), (2) clinical procedures (whether flap was elevated, number and condition of socket walls, grafting material used, application of a barrier/membrane, and follow-up time), and (3) outcomes of interest (changes in buccolingual width and midbuccal, midlingual, mesial, and distal height). Other variables recorded for subgroup analyses were flap elevation, whether a barrier was used in the experimental group, and type of grafting material applied. Corresponding authors of 5 studies were contacted for further information regarding study design or missing data to make a decision for inclusion and/or to utilize data specific to nonmolar teeth (Serino et al., 2003; Kerr et al., 2008; Azizi and Moghadam, 2009; Barone et al., 2013; Cardaropoli et al., 2012).

\section{Data Analyses and Assessment of Heterogeneity and Bias}

For the quantitative analyses, data from the selected studies were combined to estimate the effect size, defined as the difference in mean parameter change between the experimental and control groups. This was considered with respect to the 5 predetermined outcomes of interest: changes in buccolingual width and buccal, lingual, mesial, and distal height - all measured in millimeters. Random effects models were utilized throughout to estimate the effect sizes and provide $95 \%$ confidence intervals (CIs). The decision to use random effects modeling was supported not only by the clear diversity in details of the treatment protocols but by the evidence from formal statistical evaluation supporting significant heterogeneity in effect sizes (DerSimonian and Laird, 1986).

Heterogeneity of effect across the studies was formally assessed with the Cochran $Q$ test (Cochran, 1954) and characterized with the $I^{2}$ statistic, which describes the proportion of total variation in the estimated effect sizes due to the heterogeneity among studies 


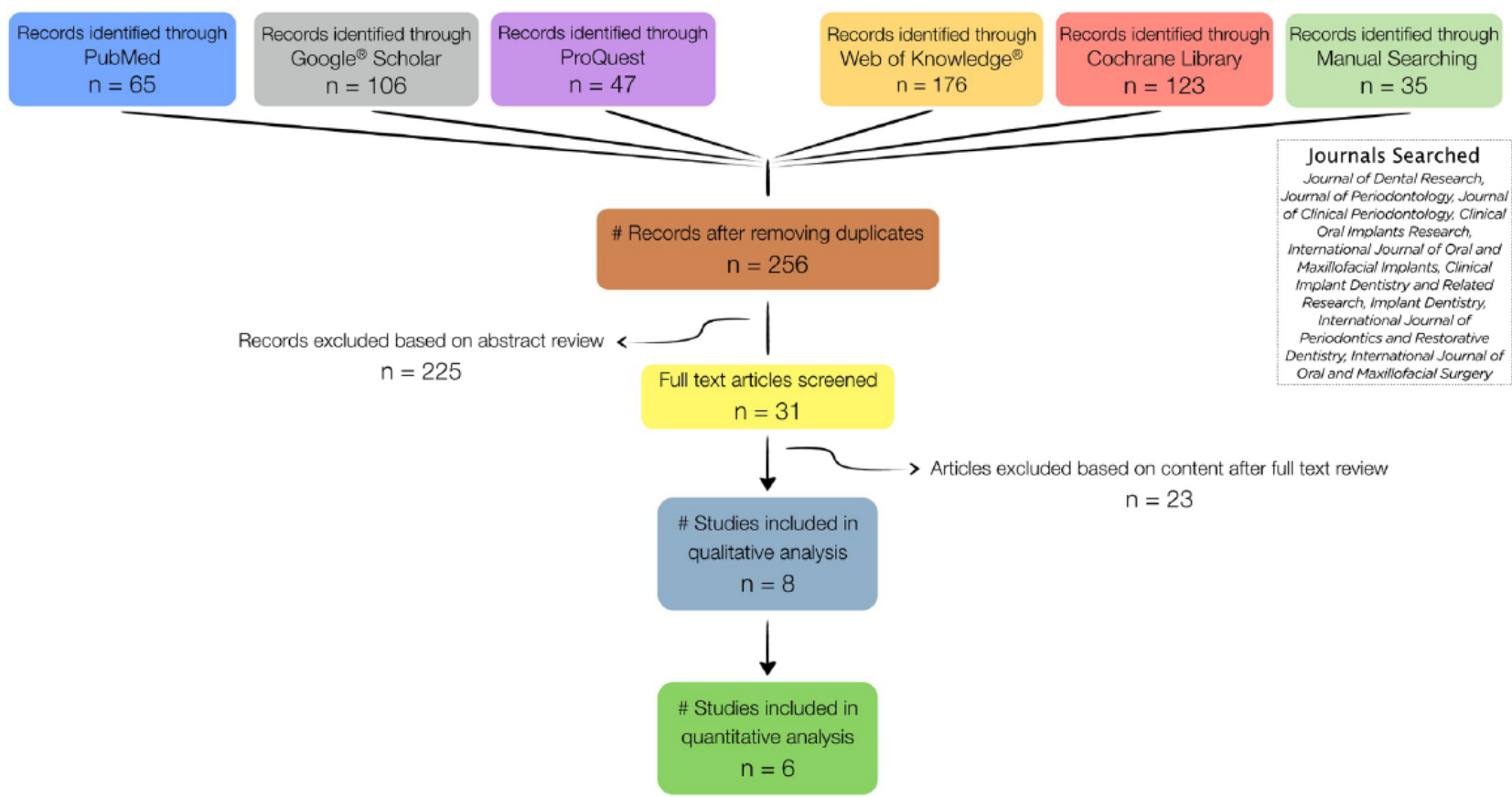

Figure 2. Flowchart depicting the search strategy and selection process.

(Higgins and Thompson, 2002). To further quantify the extent of heterogeneity among the studies, we estimated the between-studies variance ( $\tau^{2}$; Higgins et al., 2003). Forest plots were used to depict individual effect sizes as well as combined effects.

Evaluations of subgroup effects based on aspects of the experimental protocol were also undertaken to explore the potential impact of protocol variation. Three factors were considered for the subgroup analyses: whether the flap was elevated as part of the intervention, whether a barrier membrane was used, and what type of bone graft was used (xenograft, alloplast, or allograft). If status for a particular factor was not reported, a "not reported" category was used in the subgroup analysis. Differences in effect size associated with subgroups were formally assessed with respect to each of the 5 ridge measurements of interest (Borenstein et al., 2009). Estimated effect sizes and $95 \%$ CIs based on individual studies and combined analyses were displayed with forest plots. Risk of bias of the included RCTs was assessed according to Higgins et al. (2011). Potential publication bias for the studies included in the quantitative analyses was assessed via the funnel plot approach. Statistical analyses and funnel plots were performed with statistical software (Comprehensive Meta-Analysis Version 2; Biostat Inc., Englewood, NJ, USA), and forest plots were constructed with another software package (Stata Statistical Software Release 12; StataCorp LP, College Station, TX, USA).

\section{RESULTS}

\section{Study Selection}

The initial search in 5 databases yielded a total 552 records. After duplicates and nonpertinent articles were eliminated, a total of 31 articles were left for full-text assessment. Based on the predetermined eligibility criteria, a final selection of 8 RCTs for qualitative analysis was made (Camargo et al., 2000; Iasella et al., 2003; Barone et al., 2008, 2013; Aimetti et al., 2009; Azizi and Moghadam, 2009; Festa et al., 2011; Cardaropoli et al., 2012). Several studies were excluded for more than one reason. Reasons for exclusion were as follows: control group did not meet the inclusion criteria (Mardas et al., 2010; Oghli and Steveling, 2010; Mardas et al., 2011); molar teeth were included (Kerr et al., 2008; Casado et al., 2010); no bone graft was used in the experimental group (Lekovic et al., 1997, 1998; Yilmaz et al., 1998; Serino et al., 2003; Bianchi et al., 2004; Fiorellini et al., 2005; Kerr et al., 2008; Oghli and Steveling, 2010; Pelegrine et al., 2010); soft tissue grafting was performed simultaneously (Hu et al., 2009); immediate denture was delivered postextraction (Quinn et al., 1985; Kwon et al., 1986; Hoad-Reddick et al., 1994; Bolouri et al., 2001); outcomes of interest were not reported (Hoad-Reddick et al., 1994; Bolouri et al., 2001; Nevins et al., 2006; Crespi et al., 2009; Barone et al., 2012; Brownfield and Weltman, 2012; Heinemann et al., 2012); follow-up time was $<12$ wk (Nevins et al., 2006; Heinemann et al., 2012); and study was not on the topic of ridge preservation postextraction (Sisti et al., 2012). Two selected RCTs had a split-mouth design, which made pooled metaanalysis with parallel arms studies not feasible; hence, they were excluded from the quantitative analysis (Camargo et al., 2000; Festa et al., 2011). The search and selection flowchart is displayed in Figure 2. Characteristics and risk of bias assessment of the selected studies are in Appendix Tables 1 and 2, respectively.

\section{Synthesis of Results from Meta-analyses}

Data from the 6 studies eligible for quantitative analysis (Table 1) were combined to estimate the effect size of all the parameters of interest (Iasella et al., 2003; Aimetti et al., 2009; Azizi and 
Table 1. Outcomes of Interest Reported in the Included Studies: Mean Change

\begin{tabular}{|c|c|c|c|c|c|c|c|c|c|c|}
\hline \multirow[b]{3}{*}{ Author Year } & \multicolumn{5}{|c|}{ Control Group } & \multicolumn{5}{|c|}{ Experimental Group } \\
\hline & \multirow[b]{2}{*}{ Horizontal $^{a}$} & \multicolumn{4}{|c|}{ Vertical } & \multirow[b]{2}{*}{ Horizontal $^{a}$} & \multicolumn{4}{|c|}{ Vertical } \\
\hline & & MB & ML & Mesial & Distal & & $M B$ & ML & Mesial & Distal \\
\hline Aimetti 2009 & $-3.2 \pm 1.8$ & $-1.2 \pm 0.6$ & $-0.9 \pm 1.1$ & $-0.5 \pm 0.9$ & $-0.5 \pm 1.1$ & $-2.0 \pm 1.1$ & $-0.5 \pm 1.1$ & $-0.7 \pm 0.6$ & $-0.2 \pm 0.6$ & $-0.4 \pm 0.9$ \\
\hline Azizi 2010 & $-4.1 \pm 0.6$ & $-4.2 \pm 1.5$ & $-2.8 \pm 1.4$ & $-0.3 \pm 1.1$ & $-0.4 \pm 1.0$ & $-2.6 \pm 1.2$ & $-0.9 \pm 1.4$ & $-0.3 \pm 1.1$ & $-0.1 \pm 0.7$ & $-0.3 \pm 0.8$ \\
\hline Barone 2008 & $-4.5 \pm 0.8$ & $-3.6 \pm 1.5$ & $-3.0 \pm 1.6$ & $-0.4 \pm 1.2$ & $-0.5 \pm 1.0$ & $-2.5 \pm 1.2$ & $-0.7 \pm 1.4$ & $-0.4 \pm 1.3$ & $-0.2 \pm 0.8$ & $-0.4 \pm 0.8$ \\
\hline Barone 2012 & $-3.1 \pm 0.4$ & $-2.3 \pm 0.5$ & $-2.1 \pm 0.8$ & $-1.5 \pm 0.8$ & $-1.1 \pm 0.8$ & $-1.3 \pm 0.5$ & $-1.6 \pm 0.9$ & $-1.8 \pm 0.5$ & $-0.6 \pm 0.5$ & $-1.0 \pm 0.9$ \\
\hline Camargo 2000 & $-3.0 \pm 2.4$ & $-1 \pm 2.25$ & $N R^{*}$ & $N R^{*}$ & $N R^{*}$ & $-3.48 \pm 2.68$ & $-0.38 \pm 3.18$ & $N R^{*}$ & $N R^{*}$ & $N R^{*}$ \\
\hline Cardaropoli 2012 & $-4.4 \pm 0.7$ & $-1.8 \pm 0.3$ & $N R^{*}$ & $N R^{*}$ & $N R^{*}$ & $-1.1 \pm 1.1$ & $1.1 \pm 0.8$ & $N R^{*}$ & $N R^{*}$ & $N R^{*}$ \\
\hline $\begin{array}{l}\text { Festa } 2011 \\
\text { lasella } 2003\end{array}$ & $\begin{array}{l}-3.7 \pm 1.2 \\
-2.6 \pm 2.3\end{array}$ & $\begin{array}{l}-3.1 \pm 1.3 \\
-0.9 \pm 1.6\end{array}$ & $\begin{array}{l}-2.4 \pm 1.6 \\
-0.4 \pm 1.0\end{array}$ & $\begin{array}{l}-0.4 \pm 1.2 \\
-1.0 \pm 0.8\end{array}$ & $\begin{array}{l}-0.5 \pm 1 \\
-0.8 \pm 0.8\end{array}$ & $\begin{array}{l}-1.8 \pm 1.3 \\
-1.2 \pm 0.9\end{array}$ & $\begin{array}{r}-0.6 \pm 1.4 \\
1.3 \pm 2.0\end{array}$ & $\begin{array}{r}-0.5 \pm 1.3 \\
0.0 \pm 1.3\end{array}$ & $\begin{array}{l}-0.3 \pm 0.8 \\
-0.1 \pm 0.7\end{array}$ & $\begin{array}{l}-0.4 \pm 0.8 \\
-0.1 \pm 0.7\end{array}$ \\
\hline
\end{tabular}

All values are expressed in millimeters, mean $\pm \mathrm{SD}$.

$M B$, midbuccal; $M L$, midlingual; $N R$, not reported.

aBuccolingual.

Table 2. Results of Meta-analysis Assessing the Effect of Experimental Treatment vs. Control Based on the Mean Difference in Change on Alveolar Bone Measurements and Utilizing a Random Effects Model

\begin{tabular}{|c|c|c|c|c|c|c|}
\hline \multirow[b]{2}{*}{ Outcome } & \multirow[b]{2}{*}{ Mean Difference ${ }^{a}$} & \multirow{2}{*}{$\frac{\text { Treatment Effect Test }}{p \text { Value }^{b}}$} & \multicolumn{2}{|c|}{ Heterogeneity Test } & \multirow[b]{2}{*}{$1^{2}, \% \mathrm{e}$} & \multirow[b]{2}{*}{$\mathrm{Tau}^{f}$} \\
\hline & & & $Q$ Value $^{c}(d f)$ & $p$ Value $^{d}$ & & \\
\hline Buccolingual & $1.89(1.41,2.36)$ & $<.001$ & $12.28(5)$ & .031 & 59.3 & 0.44 \\
\hline Midbuccal & $2.07(1.03,3.12)$ & $<.001$ & $48.83(5)$ & $<.001$ & 89.8 & 1.22 \\
\hline Midlingual & $1.18(0.17,2.19)$ & .022 & $35.77(4)$ & $<.001$ & 88.8 & 1.08 \\
\hline Mesial & $0.48(0.18,0.79)$ & .002 & $5.33(4)$ & .26 & 24.9 & 0.17 \\
\hline Distal & $0.24(-0.05,0.53)$ & .102 & $2.92(4)$ & .57 & 0.0 & 0.0 \\
\hline
\end{tabular}

The effect size estimated is the difference (treatment - control) in mean change in the specified alveolar bone measurement and represents the gain associated with treatment. Values in millimeters, mean ( $95 \%$ confidence interval).

${ }^{b}$ Significance probability associated with the test of a significant treatment effect.

'Test statistic and degrees of freedom associated with the Cochran $Q$ test of heterogeneity of treatment effect among studies.

dSignificance probability associated with the Cochran $Q$ test of heterogeneity of treatment effect among studies.

eEstimated proportion of total variation across studies that is due to heterogeneity.

Éstimate of the standard deviation of underlying effects across studies, reflecting variability among effect sizes.

Moghadam, 2009; Barone et al., 2008, 2013; Cardaropoli et al., 2012). Results of the meta-analysis are displayed in Table 2.

\section{Buccolingual Width Changes}

Quantitative analyses revealed a strong positive ridge preservation effect in favor of the experimental group (ARP via socket grafting) of $1.89 \mathrm{~mm}(95 \% \mathrm{CI}: 1.41,2.36$; $p$ for test of treatment effect $<.001$; heterogeneity: $I^{2}=59.3 \% ; \tau=0.44$ ) as illustrated in Figure 3A.

\section{Midbuccal Height Changes}

Quantitative analyses revealed a strong positive ridge preservation effect in favor of the experimental group (ARP via socket grafting) of $2.07 \mathrm{~mm}$ (95\% CI: 1.03, 3.12; $p$ for test of treatment effect $<.001$; heterogeneity: $I^{2}=89.8 \% ; \tau=1.22$ ) as illustrated in Figure 3B.

\section{Midlingual Height Changes}

Quantitative analyses revealed a positive ridge preservation effect in favor of the experimental group (ARP via socket grafting) of $1.18 \mathrm{~mm}(95 \% \mathrm{CI}: 0.17,2.19 ; p$ for test of treatment effect $=.022$; heterogeneity: $I^{2}=88.8 \% ; \tau=1.08$ ), as illustrated in Figure 3C.

\section{Mesial Height Changes}

Quantitative analyses revealed a positive ridge preservation effect in favor of the experimental group (ARP via socket grafting) of $0.48 \mathrm{~mm}(95 \% \mathrm{CI}: 0.18,0.79 ; p$ for test of treatment effect $=.002$; heterogeneity: $I^{2}=24.9 \% ; \tau=0.17$ ), as illustrated in Figure 3D.

\section{Distal Height Changes}

Quantitative analyses revealed a positive ridge preservation effect in favor of the experimental group (ARP via socket grafting) of $0.24 \mathrm{~mm}(95 \% \mathrm{CI}:-0.05,0.53 ; p$ for test of treatment effect $=.102$; heterogeneity: $I^{2}=0 \% ; \tau=0$ ) but did not achieve statistical significance, as illustrated in Figure $3 \mathrm{E}$.

\section{Subgroup Analyses}

Table 3 displays results of subgroup analyses exploring possible differences in treatment effect based on variations in the experimental treatment protocol (i.e., whether flap elevation was 
A

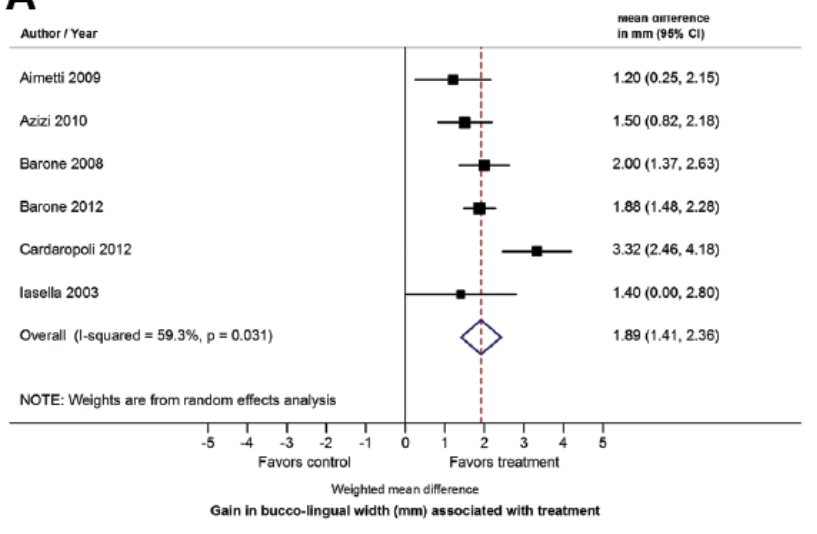

C

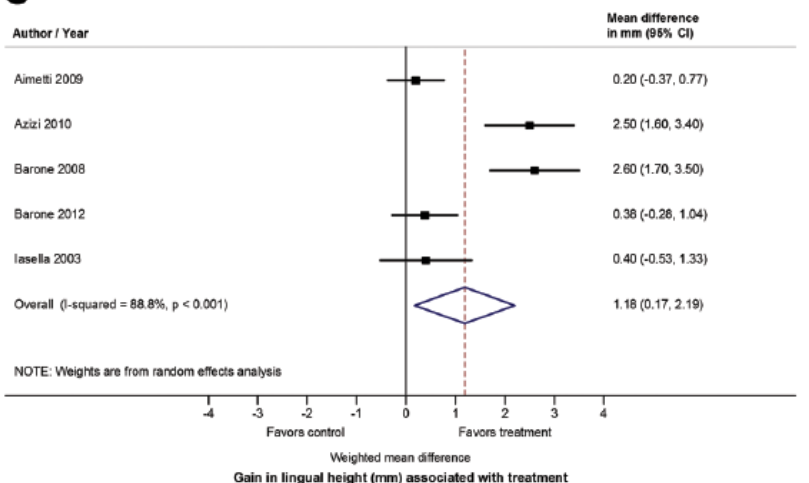

B

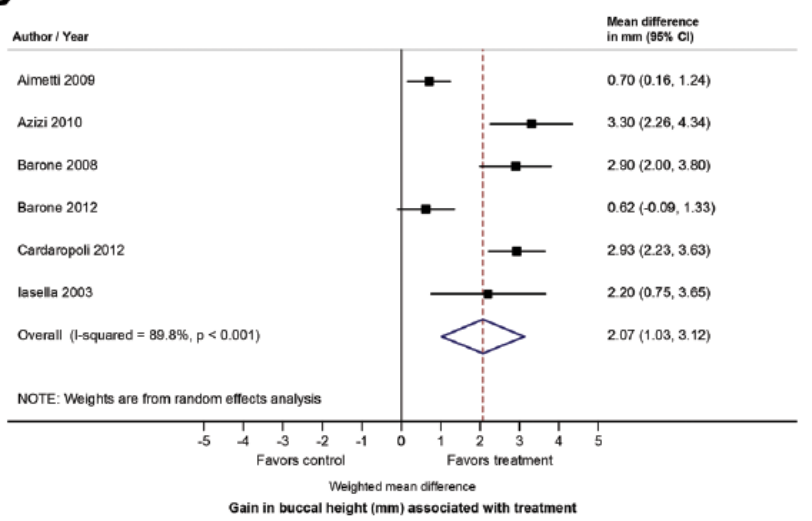

D

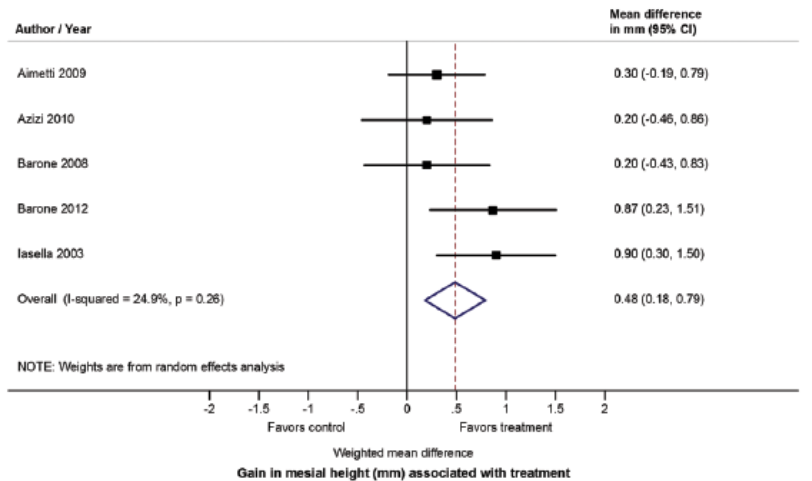

E

Barone 2008

Barone 2012

laselas 2003

Overall (1.squared $=0.0 \%, p=0.57$ )

NOTE: Weights are from random effects analysis

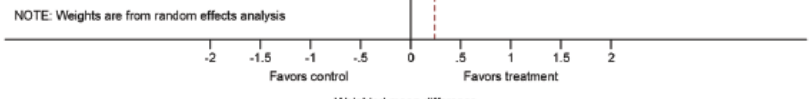

Weignted mean difterence

Gin in dibto helght (mm) associtod with troatmont

$0.13(-0.73,0.99)$

$0.70(0.10,1.30)$

$0.24(-0.05,0.52)$

Figure 3. Forest plots showing differences in buccolingual width (A), midbuccal height (B), midlingual height (C), mesial height (D), and distal height (E) changes between groups.

performed, integrity of extraction socket, whether a barrier membrane was used, and type of bone grafting material employed). Of the 15 assessments, 3 were significant at the nominal .05 level, while 2 suggested a correlation $(.05<p<$ $.10)$. Details for all significant and suggestive results are presented below.

\section{Flap Elevation}

Metaregression analyses revealed that flap elevation could have a beneficial effect on preservation of midbuccal $(p=.059)$ and midlingual $(p=.035)$ alveolar bone height. Corresponding forest plots are in Appendix Figures 1 and 2.

\section{Use of a Barrier/Membrane}

Among the experimental sites, the use of a membrane had a strong beneficial effect on preservation of midbuccal $(p=.008)$ and midlingual $(p=.067)$ alveolar bone height. Corresponding forest plots are in Appendix Figures 3 and 4.

\section{Type of Bone-Grafting Material}

Subgroup analyses revealed that the use of a xenograft or an allograft had a beneficial effect in midbuccal alveolar bone height preservation as compared to alloplastic materials $(p=$ .017). Forest plot is in Appendix Figure 5. 
Table 3. Results of Subgroup Analyses Exploring Possible Differences in Treatment Effect Based on Treatment Protocol Variations: Horizontal and Vertical Change, $p$ Values

Test of Subgroup Heterogeneity ${ }^{b}$ for 5 Outcomes of Interest

\begin{tabular}{lccccc}
\cline { 2 - 5 } Factor $^{a}$ & $\begin{array}{c}\text { Buccolingual } \\
\text { Horizontal }\end{array}$ & Midbuccal Vertical & Midlingual Vertical & Distal Vertical & Mesial Vertical \\
\hline Flap elevation & .505 & $.059^{\mathrm{c}}$ & $.035^{\mathrm{d}}$ & .56 & .797 \\
Use of a barrier membrane & .131 & $.008^{\mathrm{d}}$ & $.067^{\mathrm{c}}$ & .621 & .415 \\
Type of bone graft used & .235 & $.017^{\mathrm{d}}$ & .159 & .232 & .286 \\
\hline
\end{tabular}

aFactor upon which subgroup analysis was based.

${ }^{b}$ Significance probability associated with the test of the null hypothesis that subgroups defined by the specified moderating factor did not differ in treatment effect.

${ }^{c} .05<p<.10$; possible suggestive result.

${ }^{d} p \leq .05$; however, result did not remain significant after adjustment for multiple comparisons.

\section{DISCUSSION}

As dentistry evolves into a modern era, research aimed at further understanding the biological processes underlying alveolar bone healing, osseointegration, and tissue augmentation procedures is critical to develop predictable and successful dental implant therapy protocols with the ultimate goal of providing highquality patient care (Berglundh and Giannobile, 2013). Over the past 2 decades, numerous tissue augmentation techniques (hard and soft) have been proposed to re-create missing structures that would facilitate implant placement, as well as ridge preservation approaches to minimize bone loss after tooth extraction (McAllister and Haghighat, 2007). However, the indication of specific approaches to achieve a predictable and satisfactory outcome in a given scenario remains a significant challenge in clinical practice. In our systematic review, we made a purposeful attempt to maximize the clinical relevance of the reported results by selecting only studies with a well-defined clinical protocol (ARP via socket grafting with or without a barrier) in a particular scenario (single-tooth extraction of nonmolar teeth) that likely represents the most commonly indicated approach for ARP in contemporary dental practice (Horowitz et al., 2012).

Interestingly, several split-mouth RCTs have reported information demonstrating that the magnitude of ridge alterations after tooth extraction varies not only between but also within subjects (Camargo et al., 2000; Serino et al., 2003; Crespi et al., 2009; Festa et al., 2011). This is suggestive of the role that individual systemic and local characteristics may play on the rate, extent, and timing of ridge resorption. A plethora of factors may influence ridge resorption patterns - such as the number of neighboring teeth to be extracted, socket morphology (i.e., single- vs. multirooted teeth and socket integrity), periodontal biotype (i.e., bony buccal plate and soft tissue thickness), grafting material, smoking status, systemic factors (e.g., uncontrolled diabetes, bone metabolic disorders), and patient compliance. Therefore, eligibility criteria were carefully established to avoid the influence of some of these factors in study outcomes. For example, molars were excluded since the size and morphology of the sockets are quite different from those of single-rooted teeth and, therefore, the healing processes are not comparable.

\section{Main Findings}

We found that ARP is effective in limiting physiologic ridge reduction as compared with tooth extraction alone. The clinical magnitude of the effect was $1.89 \mathrm{~mm}(95 \%$ CI: 1.41, 2.36; $p<$ $.001)$ in terms of buccolingual width, $2.07 \mathrm{~mm}(95 \% \mathrm{CI}$ : 1.03 , 3.12; $p<.001)$ for midbuccal height, $1.18 \mathrm{~mm}(95 \%$ CI: 0.17 , $2.19 ; p=.022)$ for midlingual height, $0.48 \mathrm{~mm}(95 \% \mathrm{CI}: 0.18$, $0.79 ; p=.002)$ for mesial height, and $0.24 \mathrm{~mm}(95 \% \mathrm{CI}:-0.05$, $0.53 ; p=.102$ ) for distal height changes. Hence, strong evidence for a significant treatment effect was found for changes in buccolingual width, midbuccal height, and midlingual height. There was also evidence of a beneficial effect for mesial height, but it was not as pronounced. There was no evidence of a significant treatment effect for distal height. Interestingly, $I^{2}$ values demonstrate a strong evidence for heterogeneity of effect size for buccolingual width, midbuccal height, and midlingual height but not for distal height or mesial height (Table 2). In relation to this, it is important to remark that in one of the studies included in the quantitative analysis, a net increase of approximately $1.1 \mathrm{~mm}$ in midbuccal height was observed in the experimental group (Cardaropoli et al., 2012). This can be considered an unusual outcome, possibly explained by measurement errors owing to the absence of examiner calibration and the fact that it was the only included study that assessed therapy outcomes based on stone cast measurements, which may have errors due to the inclusion of the soft tissue in the dimensional measurements.

Our buccolingual width findings are in agreement with other meta-analyses of similar design on this topic. Vignoletti et al. (2012) reported a significant reduction of bone width when ARP was performed: $1.83 \mathrm{~mm}(95 \% \mathrm{CI}:-2.947,-0.79 ; p<.001)$. However, it should be mentioned that the results from 10 studies that followed diverse clinical protocols in noncomparable clinical scenarios were pooled, which may have had a significant influence in the results. Vittorini Orgeas et al. (2013) performed a meta-analysis distinguishing among multiple clinical ridge preservation approaches. They found that in sites that received bone grafting and a barrier-which represents the protocol followed in the majority of the studies included in our quantitative analysis (5 of 6) - the weighed benefit of ARP was $1.99 \mathrm{~mm}$ 
(95\% CI: $-0.086,2.485 ; p=.06)$. In terms of height changes, Vignoletti et al. observed a beneficial effect of $1.47 \mathrm{~mm}(95 \%$ CI: $-1.982,-0.953 ; p<.001)$ when ARP was performed as compared to a control. Vittorini Orgeas et al. found a nonsignificant difference of $0.96 \mathrm{~mm}(95 \% \mathrm{CI}:-1.177,3.101 ; p=.37)$ in terms of height change differences between ARP via bone grafting and a membrane as compared with controls, in favor of the former. This observation may be explained on the basis that only 3 studies, which reported quite divergent findings, were considered in this category (Camargo et al., 2000; Iasella et al., 2003; Barone et al., 2008). However, a comparison of these height changes with those reported in this review does not appear to be reasonable, since a specific distinction among midbuccal, midlingual, mesial, and distal height changes was not made in previous systematic reviews. As mentioned in previous systematic reviews (Ten Heggeler et al., 2011; Vignoletti et al., 2012; Morjaria et al., 2014), although a benefit was observed in association with ARP, some degree of horizontal and vertical bone loss should still be generally expected (Table 1).

\section{Subgroup Analyses}

Subgroup analyses were conducted to assess the influence of flap elevation, usage of a membrane to cover the socket, and type of bone graft on the observed effect sizes for each clinical outcome. Of the 15 assessments, 3 were significant $(p \leq .05)$, while 2 of them were suggestive $(.05<p<.10)$. Buccolingual width and mesial and distal height changes were not influenced by variations on flap elevation, membrane usage, and type of bone graft. Sites that underwent flap elevation exhibited less average midbuccal $(p=.059)$ and midlingual $(p=.035)$ height loss (Appendix Figures 1 and 2). There were 3 studies on each subgroup: flap elevation (Iasella et al., 2003; Barone et al., 2008; Azizi and Moghadam, 2009) and no flap elevation (Aimetti et al., 2009; Barone et al., 2013; Cardaropoli et al., 2012). This is an interesting result: although it is generally acknowledged that flap elevation has a detrimental impact in bone remodeling because of the interruption of the periosteal vascular supply and an increase in postsurgical local inflammation, recent preclinical evidence indicates that flap elevation does not promote alveolar bone loss (Araujo and Lindhe, 2009; Fickl et al., 2011). The usage of a membrane was strongly associated with less average midbuccal height loss $(p=.008)$ and midlingual height loss ( $p=.067$; Appendix Figures 3 and 4). There were 5 studies in the membrane subgroup (Iasella et al., 2003; Barone et al., 2008, 2013; Azizi and Moghadam, 2009; Cardaropoli et al., 2012), and only 1 did not report the use of a membrane (Aimetti et al., 2009), which may have had an influence in the observed results; however, these findings are aligned with current evidence. Interestingly, sites grafted with a xenograft (Barone et al., 2008, 2013; Azizi and Moghadam, 2009; Cardaropoli et al., 2012) or an allograft (Iasella et al., 2003) exhibited less midbuccal height loss $(p=.017)$ as compared with sites that received an alloplast (Aimetti et al., 2009), as shown in Appendix Figure 5. To our knowledge, this is the first study that reports a subanalysis to determine the effect of the biomaterial used on clinical outcomes of interest.

\section{Limitations}

Although we utilized a comprehensive search strategy to identify articles in the gray literature, it is possible that some gray literature was not included, because only 1 database containing this type of information was searched. Although the total number of subjects enrolled in the selected studies can be considered sufficient for the assessment of effect size differences between groups for the main outcomes of interest, it is important to remark that the reported subgroup analysis results should be taken with caution, given the limited number of studies $(n=6)$ that were eligible for the quantitative analysis. Also, the assessment method used in all the included studies (i.e., nonstandardized linear measurements) does not necessarily provide critical information on bone availability for implant placement planning. This stresses the importance of developing and applying research protocols that would allow a precise and objective quantification of the total tridimensional changes after tooth extraction (Chappuis et al., 2013) in future clinical trials.

\section{CONCLUSIONS}

The results of this systematic review and subsequent metaanalysis showed that ARP via socket filling with a bone graft can be an effective therapy to prevent physiologic bone loss after extraction of nonmolar teeth, in both the horizontal and the vertical dimension. Subgroup analyses showed that flap elevation, the usage of a membrane, and the application of a xenograft or an allograft may contribute to enhance the outcomes, particularly on midbuccal and midlingual height preservation.

Nevertheless, a certain degree of ridge volume loss should be expected even if ARP is applied. Hence, ARP may significantly prevent alveolar bone remodeling postextraction, but this effect is variable, likely due to the influence of local and systemic factors that are not fully understood yet. While in certain cases ARP could result in the maintenance of sufficient bone volume to place an implant in an ideal restorative position without the need of ancillary implant site development procedures, it may not be sufficient in other clinical instances. This information is expected to be of use not only to clinicians and patients but also to policy makers, given the apparently robust beneficial effect that ARP may provide in the specific scenario addressed in this review.

\section{ACKNOWLEDGMENTS}

The authors thank Ms. Christine White, specialist librarian at the University of Iowa College of Dentistry, for her assistance in the conduction of the search. We also express our gratitude to the authors who provided additional information: Dr. Azizi (University of Isfahan, Iran), Dr. Barone (University of Pisa, Italy), Dr. Camargo (University of California, Los Angeles, USA), Dr. Cardaropoli (private practice, Torino, Italy), Dr. Mealey (University of Texas Health Science Center at San Antonio, USA), and Dr. Serino (private practice, Rome, Italy). Finally, G.A.-O. and S.E. acknowledge the American Academy of Periodontology Foundation for the support provided to pursue of a career in academia. The author(s) received no financial 
support and declare no potential conflicts of interest with respect to the authorship and/or publication of this article.

\section{REFERENCES}

Aimetti M, Romano F, Griga FB, Godio L (2009). Clinical and histologic healing of human extraction sockets filled with calcium sulfate. Int $J$ Oral Maxillofac Implants 24:902-909.

Araujo MG, Lindhe J (2009). Ridge alterations following tooth extraction with and without flap elevation: an experimental study in the dog. Clin Oral Implants Res 20:545-549.

Artzi Z, Nemcovsky CE (1998). The application of deproteinized bovine bone mineral for ridge preservation prior to implantation: clinical and histological observations in a case report. Journal of periodontology 69:1062-1067.

Azizi A, Moghadam SA (2009). A study on the effect of Bio-Oss and collagen membrane on the repair of dental socket. Journal of Isfahan Dental School 5:133-139.

Barone A, Aldini NN, Fini M, Giardino R, Calvo Guirado JL, Covani U (2008). Xenograft versus extraction alone for ridge preservation after tooth removal: a clinical and histomorphometric study. J Periodontol 79:1370-1377.

Barone A, Orlando B, Cingano L, Marconcini S, Derchi G, Covani U (2012). A randomized clinical trial to evaluate and compare implants placed in augmented versus non-augmented extraction sockets: 3-year results. $J$ Periodontol 83:836-846.

Barone A, Ricci M, Tonelli P, Santini S, Covani U (2013). Tissue changes of extraction sockets in humans: a comparison of spontaneous healing vs. ridge preservation with secondary soft tissue healing. Clin Oral Implants Res 24:1231-1237.

Berglundh T, Giannobile WV (2013). Investigational clinical research in implant dentistry: beyond observational and descriptive studies. $J$ Dent Res 92(12):107S-108S.

Bianchi J, Fiorellini JP, Howell TH, Sekler J, Curtin H, Nevins ML, et al. (2004). Measuring the efficacy of rhBMP-2 to regenerate bone: a radiographic study using a commercially available software program. Int $J$ Periodontics Restorative Dent 24:579-587.

Bolouri A, Haghighat N, Frederiksen N (2001). Evaluation of the effect of immediate grafting of mandibular postextraction sockets with synthetic bone. Compend Contin Educ Dent 22:955-958, 960, 962 passim; quiz 966.

Borenstein M, Hedges LV, Higgins JP, Rothstein H (2009). Subgroup analyses. In: Introduction to Meta-analysis; Borenstein M, editor. Chichester, UK: Wiley, pp 149-186.

Brownfield LA, Weltman RL (2012). Ridge preservation with or without an osteoinductive allograft: a clinical, radiographic, micro-computed tomography, and histologic study evaluating dimensional changes and new bone formation of the alveolar ridge. J Periodontol 83:581-589.

Camargo PM, Lekovic V, Weinlaender M, Klokkevold PR, Kenney EB, Dimitrijevic B, et al. (2000). Influence of bioactive glass on changes in alveolar process dimensions after exodontia. Oral Surg Oral Med Oral Pathol Oral Radiol Endod 90:581-586.

Cardaropoli D, Tamagnone L, Roffredo A, Gaveglio L, Cardaropoli G (2012). Socket preservation using bovine bone mineral and collagen membrane: a randomized controlled clinical trial with histologic analysis. Int J Periodontics Restorative Dent 32:421-430.

Casado PL, Duarte ME, Carvalho W, Esmeraldo da Silva L, Barboza EP (2010). Ridge bone maintenance in human after extraction. Implant Dent 19:314-322.

Chappuis V, Engel O, Reyes M, Shahim K, Nolte LP, Buser D (2013). Ridge alterations post-extraction in the esthetic zone: a $3 \mathrm{D}$ analysis with CBCT. J Dent Res 92(12):195S-201S.

Christensen GJ (1996). Ridge preservation: why not? J Am Dent Assoc 127:669-670.

Cochran WG (1954). The combination of estimates from different experiments. Biometrics 10:101-129.

Crespi R, Capparè P P, Gherlone E (2009). Magnesium-enriched hydroxyapatite compared to calcium sulfate in the healing of human extraction sockets: radiographic and histomorphometric evaluation at 3 months. $J$ Periodontol 80:210-218

Darby I, Chen ST, Buser D (2009). Ridge preservation techniques for implant therapy. Int J Oral Maxillofac Implants 24(Suppl):260-271.

De Buitrago JG, Avila-Ortiz G, Elangovan S (2013). Quality assessment of systematic reviews on alveolar ridge preservation. $J$ Am Dent Assoc 144:1349-1357

DerSimonian R, Laird N (1986). Meta-analysis in clinical trials. Control Clin Trials 7:177-188.

Festa VM, Addabbo F, Laino L, Femiano F, Rullo R (2011). Porcine-derived xenograft combined with a soft cortical membrane versus extraction alone for implant site development: a clinical study in humans. Clin Implant Dent Relat Res 15:707-713.

Fickl S, Kebschull M, Schupbach P, Zuhr O, Schlagenhauf U, Hurzeler MB (2011). Bone loss after full-thickness and partial-thickness flap elevation. J Clin Periodontol 38:157-162.

Fiorellini JP, Howell TH, Cochran D, Malmquist J, Lilly LC, Spagnoli D, et al. (2005). Randomized study evaluating recombinant human bone morphogenetic protein-2 for extraction socket augmentation. $J$ Periodontol 76:605-613.

Gerritsen AE, Allen PF, Witter DJ, Bronkhorst EM, Creugers NH (2010). Tooth loss and oral health-related quality of life: a systematic review and meta-analysis. Health Qual Life Outcomes 8:126.

Hammerle CH, Araújo MG, Simion M; Osteology Consensus Group 2011 (2012). Evidence-based knowledge on the biology and treatment of extraction sockets. Clin Oral Implants Res 23(Suppl 5):80-82. (Published erratum in: Clin Oral Implants Res 23:641, 2012).

Heinemann F, Hasan I, Schwahn C, Bourauel C, Mundt T (2012). Bone level change of extraction sockets with Bio-Oss collagen and implant placement: a clinical study. Ann Anat 194:508-512.

Higgins JP, Thompson SG (2002). Quantifying heterogeneity in a metaanalysis. Stat Med 21:1539-1558.

Higgins JP, Thompson SG, Deeks JJ, Altman DG (2003). Measuring inconsistency in meta-analyses. BMJ 327:557-560.

Higgins JP, Altman DG, Gotzsche PC, Juni P, Moher D, Oxman AD, et al. (2011). The Cochrane Collaboration's tool for assessing risk of bias in randomised trials. BMJ 343:d5928.

Hoad-Reddick G, Grant AA, McCord JF (1994). Osseoretention? Comparative assessment of particulate hydroxyapatite inserted beneath immediate dentures. Eur J Prosthodont Restor Dent 3:61-65.

Horowitz R, Holtzclaw D, Rosen PS (2012). A review on alveolar ridge preservation following tooth extraction. J Evid Based Dent Pract 12(3 Suppl):149-160.

Horvath A, Mardas N, Mezzomo LA, Needleman IG, Donos N (2012). Alveolar ridge preservation. A systematic review. Clin Oral Investig 17:341-363.

Hu XL, Lin Y, Wang J, Wu H, Qiu LX, Zhang Y (2009). [Clinical study of tissue preservation of extraction socket with Bio-Oss collagen and acellular dermal matrix]. Zhonghua Kou Qiang Yi Xue Za Zhi 44:513-516.

Iasella JM, Greenwell H, Miller RL, Hill M, Drisko C, Bohra AA, et al. (2003). Ridge preservation with freeze-dried bone allograft and a collagen membrane compared to extraction alone for implant site development: a clinical and histologic study in humans. J Periodontol 74:990-999.

Kerr EN, Mealey BL, Noujeim ME, Lasho DJ, Nummikoski PV, Mellonig JT (2008). The effect of ultrasound on bone dimensional changes following extraction: a pilot study. J Periodontol 79:283-290.

Kwon HJ, el Deeb M, Morstad T, Waite D (1986). Alveolar ridge maintenance with hydroxylapatite ceramic cones in humans. J Oral Maxillofac Surg 44:503-508.

Lekovic V, Kenney EB, Weinlaender M, Han T, Klokkevold P, Nedic M, et al. (1997). A bone regenerative approach to alveolar ridge maintenance following tooth extraction: report of 10 cases. J Periodontol 68:563-570.

Lekovic V, Camargo PM, Klokkevold PR, Weinlaender M, Kenney EB, Dimitrijevic B, et al. (1998). Preservation of alveolar bone in extraction sockets using bioabsorbable membranes. J Periodontol 69:1044-1049.

Mardas N, Chadha V, Donos N (2010). Alveolar ridge preservation with guided bone regeneration and a synthetic bone substitute or a bovinederived xenograft: a randomized, controlled clinical trial. Clin Oral Implants Res 21:688-698. 
Mardas N, D'Aiuto F, Mezzomo L, Arzoumanidi M, Donos N (2011). Radiographic alveolar bone changes following ridge preservation with two different biomaterials. Clin Oral Implants Res 22:416-423.

McAllister BS, Haghighat K (2007). Bone augmentation techniques. J Periodontol 78:377-396.

Moher D, Liberati A, Tetzlaff J, Altman DG; PRISMA Group (2009). Preferred reporting items for systematic reviews and meta-analyses: the PRISMA statement. Ann Intern Med 151(4):264-269, W264.

Morjaria KR, Wilson R, Palmer RM (2014). Bone healing after tooth extraction with or without an intervention: a systematic review of randomized controlled trials. Clin Implant Dent Relat Res 16:1-20.

Nevins M, Camelo M, De Paoli S, Friedland B, Schenk RK, ParmaBenfenati S, et al. (2006). A study of the fate of the buccal wall of extraction sockets of teeth with prominent roots. Int $J$ Periodontics Restorative Dent 26:19-29.

Oghli AA, Steveling H (2010). Ridge preservation following tooth extraction: a comparison between atraumatic extraction and socket seal surgery. Quintessence Int 41:605-609.

Osburn RC (1974). Preservation of the alveolar ridge: a simplified technique for retaining teeth beneath removable appliances. J Indiana State Dent Assoc 53:8-11.

Pelegrine AA, da Costa CE, Correa ME, Marques JF Jr (2010). Clinical and histomorphometric evaluation of extraction sockets treated with an autologous bone marrow graft. Clin Oral Implants Res 21:535542.

Quinn JH, Kent JN, Hunter RG, Schaffer CM (1985). Preservation of the alveolar ridge with hydroxylapatite tooth root substitutes. $J$ Am Dent Assoc 110:189-193.

Schropp L, Wenzel A, Kostopoulos L, Karring T (2003). Bone healing and soft tissue contour changes following single-tooth extraction: a clinical and radiographic 12-month prospective study. Int $J$ Periodontics Restorative Dent 23:313-323.

Seibert JS, Salama H (1996). Alveolar ridge preservation and reconstruction. Periodontol 2000 11:69-84.

Serino G, Biancu S, Iezzi G, Piattelli A (2003). Ridge preservation following tooth extraction using a polylactide and polyglycolide sponge as space filler: a clinical and histological study in humans. Clin Oral Implants Res 14:651-658.

Sisti A, Canullo L, Mottola MP, Covani U, Barone A, Botticelli D (2012). Clinical evaluation of a ridge augmentation procedure for the severely resorbed alveolar socket: multicenter randomized controlled trial, preliminary results. Clin Oral Implants Res 23:526-535.

Ten Heggeler JM, Slot DE, Van der Weijden GA (2011). Effect of socket preservation therapies following tooth extraction in non-molar regions in humans: a systematic review. Clin Oral Implants Res 22:779-788.

Van der Weijden F, Dell'Acqua F, Slot DE (2009). Alveolar bone dimensional changes of post-extraction sockets in humans: a systematic review. J Clin Periodontol 36:1048-1058.

Vignoletti F, Matesanz P, Rodrigo D, Figuero E, Martin C, Sanz M (2012). Surgical protocols for ridge preservation after tooth extraction: a systematic review. Clin Oral Implants Res 23(Suppl 5):22-38.

Vittorini Orgeas G, Clementini M, De Risi V, de Sanctis M (2013). Surgical techniques for alveolar socket preservation: a systematic review. Int $J$ Oral Maxillofac Implants 28:1049-1061.

Weng D, Stock V, Schliephake H (2011). Are socket and ridge preservation techniques at the day of tooth extraction efficient in maintaining the tissues of the alveolar ridge? Eur J Oral Implantol 4(Suppl):59-66.

Yilmaz S, Efeoğlu E, Kiliç AR (1998). Alveolar ridge reconstruction and/or preservation using root form bioglass cones. J Clin Periodontol 25:832839. 